\title{
Special issue: applications, results \& future direction (EAIS 12): 2
}

\author{
José Antonio Iglesias Martínez • Igor Škrjanc
}

Published online: 11 May 2014

(c) Springer-Verlag Berlin Heidelberg 2014

The "real world" is changing and new problems are arising. Some of these new problems in the computer science area are related to the need of manage high-volume and complex non-stationary data streams.

The design of processing systems that can extract meaningful knowledge in real time from stream data is a present-day challenge. A potential solution for this challenge is offered by the evolving and incremental learning systems. These systems need to process useful information from huge amounts of data, analyse the data rapidly and efficiently extract knowledge in real time. Since these data are constantly changing, these systems need to change their behaviour, adapt their structure, self-evaluate and selfdevelop. The emerging area of Evolving and Adaptive Intelligent Systems is based on this idea.

This special issue focus on the different applications and results of these Evolving and Adaptive Intelligent Systems. Since the application areas are very diverse, the six papers presented in this special issue are very different. These interesting papers are extended versions (carefully peer reviewed) of selected papers submitted to the 2012 IEEE Evolving and Adaptive Intelligent Systems Conference (EAIS 12) which was held in Madrid, Spain.

The first paper "Enhanced Evolving Participatory Learning Fuzzy Modeling: An Application for Asset

\section{J. A. I. Martínez ( $₫)$}

Carlos III University of Madrid, Madrid, Spain

e-mail: jiglesia@inf.uc3m.es

URL: http://www.caos.inf.uc3m.es/ jiglesia/

\section{I. Škrjanc}

Faculty of Electrical Engineering, University of Ljubljana,

Ljubljana, Slovenia

e-mail: igor.skrjanc@fe.uni-lj.si

URL: http://msc.fe.uni-lj.si
Returns Volatility Forecasting” by Leandro Maciel, Fernando Gomide and Rosangela Ballini presents an enhanced version of the ePL (evolving Participatory Learning). Evolving participatory learning (ePL) modeling joins the concepts of participatory learning and evolving fuzzy systems. The proposed version is called ePL+ and it includes both a utility measure to shrink rule bases, and a variable cluster radius mechanism to improve the cluster structure. According to the results, the authors suggest that the ePL + approach is highly capable to model volatility dynamics, in a robust, flexible, and autonomous way.

The title of the second paper is "Adaptive MaximumLifetime Routing in Mobile Ad-hoc Networks Using Temporal Difference Reinforcement Learning" and the authors are Saloua Chettibi and Salim Chikhi. In this paper an adaptive maximum lifetime routing strategy for MANETs (Mobile Ad-hoc NETworks) based on Reinforcement Learning technique is proposed. In particular, Q-Learning and SARSA with and without eligibility-traces are used. The proposed approach is implemented on a specific routing protocol.

The third paper "A self-organized system improving inner topology for data sharing efficiency" by Fréderic Armetta, Mohammed Haddad, Salima Hassas and Hamamache Kheddouci, proposes a promising self-organizing ant inspired approach applied to unstructured peer-to-peer networks. The approach improves existing search algorithms related to scale-free overlay topologies by introducing mechanism that take into account the utility of information that is carried by peers.

Kazi Shah Nawaz Ripon and Tjim Torresen are the authors of the fourth paper: "Integrated job shop scheduling and layout planning: A hybrid evolutionary method for optimizing multiple objectives". This paper presents a multi-objective evolutionary method for solving job shop 
scheduling problems (JSSP). This method considers transportation delays and facility layout planning (FLP) as an integrated problem, which presents the final solutions as a pareto-optimal set. In this case, a hybrid genetic algorithm (GA) with variable neighbourhood search (VNS) is applied.

The last paper of this special issue is "Integrated temporal partitioning and partial reconfiguration techniques for design latency improvement" by Ramzi Ayadi, Bouraoui Ouni and Abdellatif Mtibaa. This paper presents a novel temporal partitioning methodology that temporally partitions a data flow graph on reconfigurable systems. The proposed approach optimizes the whole latency of the design and it is tested on several examples on the Xilinx Virtex-II pro. As the authors describe, the results show significant reduction in the design latency compared with other approaches used in this field.

Finally, the guest editors would like to thank all the authors and reviewers who contributed to this special issue. Also, we wish to thank the editors-in-chief and editorial office of the Evolving Systems Journal for their support. 